\title{
Diphtheria A Case Report-Early Diagnosis And Treatment Leads To Favourable Outcome.
}

\author{
Dr.Sunanda joshi ${ }^{1}$,Dr.Nalini Mittal, ${ }^{2}$ Dr. Tarun Kumar Ravi ${ }^{3}$ \\ ${ }^{I}$ (Senior Resident, Department of Microbiology,Babu Jagjiwan Ram Memorial Hospital, New Delhi,India) \\ ${ }^{2}$ (Consultant and Head of department,Department of Microbiology,Babu Jagjiwan Ram Memorial Hospital \\ New Delhi,India) \\ ${ }_{3}^{3}$ Consultant and Head of department Department of Paediatrics, Babu Jagjiwan Ram Memorial Hospital , \\ New Delhi,India)
}

\begin{abstract}
Diphtheria is an infectious disease caused by the toxin producing bacteria Corynebacterium diphtheria. Despite the introduction of mass immunization, outbreaks are still encountered in our country. Here we report a case of Diphtheria in a 8 year old un-immunised patient. This case shows the need to have high index of suspicion for diphtheria when managing a patient with membranous pharyngitis. We should have well equipped district laboratories and hospitals in Delhi as it will help in managing and diagnosing these cases early which can be life saving.
\end{abstract}

Keywords: Corynebacterium diphtheria, immunization, membranous pharyngitis

\section{Introduction}

Diphtheria is an infectious disease caused by the toxin producing bacteria Corynebacterium diphtheriae ${ }^{1}$. Diphtheria is a notifiable disease still large number of cases are not reported. ${ }^{2}$ Primary vaccination develops antibodies in $94-100 \%$ children ${ }^{3}$ which falls if booster doses are not given. ${ }^{4}$. Despite the introduction of mass immunization, outbreaks are still encountered in our country. Successful immunization programmes have eliminated diphtheria from developed countries. ${ }^{5,6,7}$. Diphtheria remained a public health problem in India.

Here we report a case of Diphtheria in an 8 year old un-immunised patient.

\section{Case Report}

8 year old patient was admitted in hospital with chief complaints of low grade fever with cough and cold since 4 days. She had difficulty in breathing since 1day and was feeling generally weak and lethargic. According to the mother she was un-immunised. On examination the patient was awake and conscious with a weak and thready pulse .She was febrile with a heart rate of $104 / \mathrm{min}$ and respiratory rate of $24 / \mathrm{min}$ with an audible wheeze. She was mildly dehydrated with cold peripheries. Initially patient was diagnosed as a case of wheezy lower respiratory tract infection. Patient was nebulized with salbutamol and started on Amoxicillin clavulanic acid and cefotaxime. Laboratory investigations showed $\mathrm{Hb}$. 11.1gm\%, TLC 14300/cumm, Neutrophils $70 \%$, lymphocytes $25 \%$, eosiniphils $04 \%$ and monocytes $01 \%$, Peripheral smear was reported as normocytic normochromic anaemia.

The following day condition of the patient detoriated and she developed hoarseness of voice with difficulty in swallowing and a diffuse swelling in the anterior part of neck. The patient also complained of worsening of respiratory symptoms. On examination both sides palatine tonsils were enlarged with small greyish white membrane present on the surface which bled on touch. She also had swollen tongue. Patient was treated on the line of possibility of Diphtheria.

Two throat swabs were sent for microbiological investigation which included Direct examination by Gram stain \&Albert stain and culture. Gram stain showed pleomorphic gram positive bacilli with swollen ends arranged in parallel and angular fashion(Figure1). Albert stain showed green coloured bacilli with black granules at their ends(Figure 2). Sample was inoculated on blood agar and Mc Conkeys agar. Selective media for diphtheria was not available as samples for diphtheria is rarely received in our laboratory. Patient was referred to higher centre for antidiphtheric serum(ADS) and confirmation of the diagnosis . There the patient was immediately started on ADS and was treated accordingly .She was confirmed to be positive for Diphtheria by microbiological culture and Elks gel test for toxin production. Condition of the patient improved and was later discharged from the hospital. Later patient reported in follow up in OPD,she has no complications and she recovered fully. 


\section{Discussion}

Acute infectious disease caused by Corynebacterium diphtheriae affects primarily the upper respiratory tract with the formation of greyish white pseudomembrane. Although the disease is distributed throughout the world ,it is endemic primarily in developing regions of Africa, Asia and South America . After the introduction of DPT vaccination ,the incidence has declined steadily and now it is a rare occurrence. Delay in treatment increases morbidity and mortality. When appropriate treatment has been administered on day one of illness mortality may be as low as $1 \%$. When treatment has been delayed until day four the mortality rate is $\leq 20$ fold higher.

We report this case of Diphtheria as the early diagnosis with strong clinical suspicion helped in saving a precious life .Vaccination has reduced the mortality and morbidity. However in India Diphtheria is endemic and it occurs in sporadic cases or small out breaks. It is fatal in 5-10\% cases. ${ }^{8}$ It is a vaccine preventable disease still large number of cases are reported worldwide in which India has a significant contribution. According to WHO in 2015 reported cases of Diphtheria in India are $2365^{9}$. National centre for disease control reported 7 outbreaks in India during the year 2014. ${ }^{8}$ A study carried out in Delhi in 2014 showed the persistence of diphtheria in Delhi and NCR. $23.2 \%$ were tested to be positive by culture for C. diphtheriae.$^{10}$

Diphtheria mainly affects children aged between 1 to $5 \mathrm{yr}$, however, due to good vaccine coverage, a change in age incidence has been observed to 5-15 years with more cases being reported in adults ${ }^{11}$. The reason for the persistence of the disease is poor primary immunisation coverage and missed booster doses. ${ }^{12,13}$

After 3 doses of vaccine, antibody develops in $94 \%$ to $100 \%$ of children. ${ }^{14,15}$ However without booster doses, antibody drops below protective levels. ${ }^{15,16}$ Low booster coverage influences lack of herd immunity and thereby contributing to higher attack rates among the community. Socioeconomic instability, migration, delays in implementation of vaccination and of treatment in response to cases, and lack of public education and awareness were contributing factors in various outbreaks.

\section{Conclusions}

This case shows the need to have high index of suspicion for diphtheria when managing a patient with membranous pharyngitis. We should have well equipped district laboratories and hospitals in Delhi as it will help in managing and diagnosing these cases early which can be life saving. Precautions should be taken by travellers to areas with diphtheria outbreaks and as recommenend by ACIP that travel to such areas immunisation should be up to date.Infants travelling to such areas should ideally receive 3 doses of DPT/DT before travelling. We should improve vaccination coverage in identified pockets, enhanced surveillance methods in this part of country.

\section{References}

[1]. Dikid T, Jain SK, Sharma A, Kumar A, Narain JP. Emerging \& re-emerging infection

[2]. Introduction of inactivated poliovirus vaccine into oral poliovirus vaccine-using countries. Wkly Epidemiol Rec 2003; 28: 241-250.

[3]. S in India: an overview. Indian J Med Res 2013; $138: 19-31$.

[4]. Vandelaer J, Bilous J, Nshimirimana D. The Reaching Every District (RED) approach as a way to improve immunization performance Bull World Health Organ 2007; 86-96.

[5]. Brown J, Monasch R, Bicego G, Burton A, Boerma JT. Assessment of the quality of national child immunization coverage estimates in population-based surveys. Chapel Hill, NC: Carolina Population Center; 2002.

[6]. Murray CJL, Shengelia B, Gupta N, Moussavi S, Tandon A, Thieren M. Validity of reported vaccination coverage in 45 countries. Lancet 2003; 362: 1022-1027.

[7]. Becker H. Problems of inference and proof in participant observation. Am Sociol Rev 1958; 23: 652-660.

[8]. Chamberlin TC. The method of multiple working hypotheses. Science 1965; 148: 754-769.

[9]. National health portal .available from https://www.nhp.gov.in/disease/respiratory/diphtheria

[10]. World Health Organization. Immunization, surveillance, 8. assessment and monitoring. Available from: http://www.who.int/entity/immunization_monitoring/data/incidence_series.xls

[11]. S. Bhagat, S.S. Grover, N. Gupta, R.D. Roy, and S. Khare Indian J Med Res. 2015 Oct; 142(4): 459-461

[12]. Singhal T, Lodha R, Kapil A, Jain Y, Kabra SK. Diphtheria- down but not out. Indian Pediatr 2000; 37: 728-38.

[13]. Murhekar MV, Bitragunta S. Persistence of diphtheria in 11. India. Indian J Community Med. 2011; 36:164-5.

[14]. Bitragunta S, Murhekar MV, Hutin YJ, Penumur PP, 3. Gupte MD. Persistence of Diphtheria, Hyderabad, India, 2003-2006. Emerg Infect Dis. 2008; 14:1144-6.

[15]. World Health Organization Diphtheria vaccine-WHO 12. position paper. Wkly Epidemiol Rec 2006;81:24-31

[16]. World Health Organization The immunological basis for immunization series. Module 2: diphtheria. Geneva: The Organization; 1993

[17]. Chen RT, Broome CV, Weinstein RA, Weaver R, Tsai TF.

[18]. Diphtheria in the United States, 1971-1981. Am J Public Health 1985;75:1393-7 


\section{Figure 1}

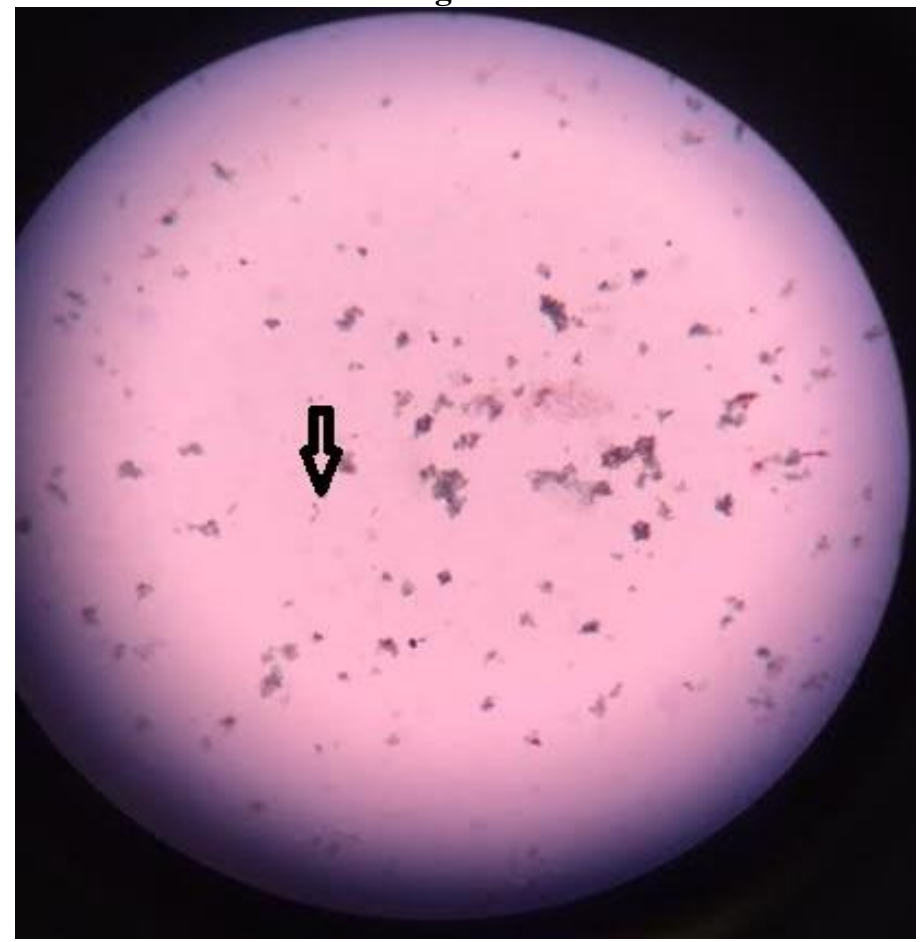

Figure 2

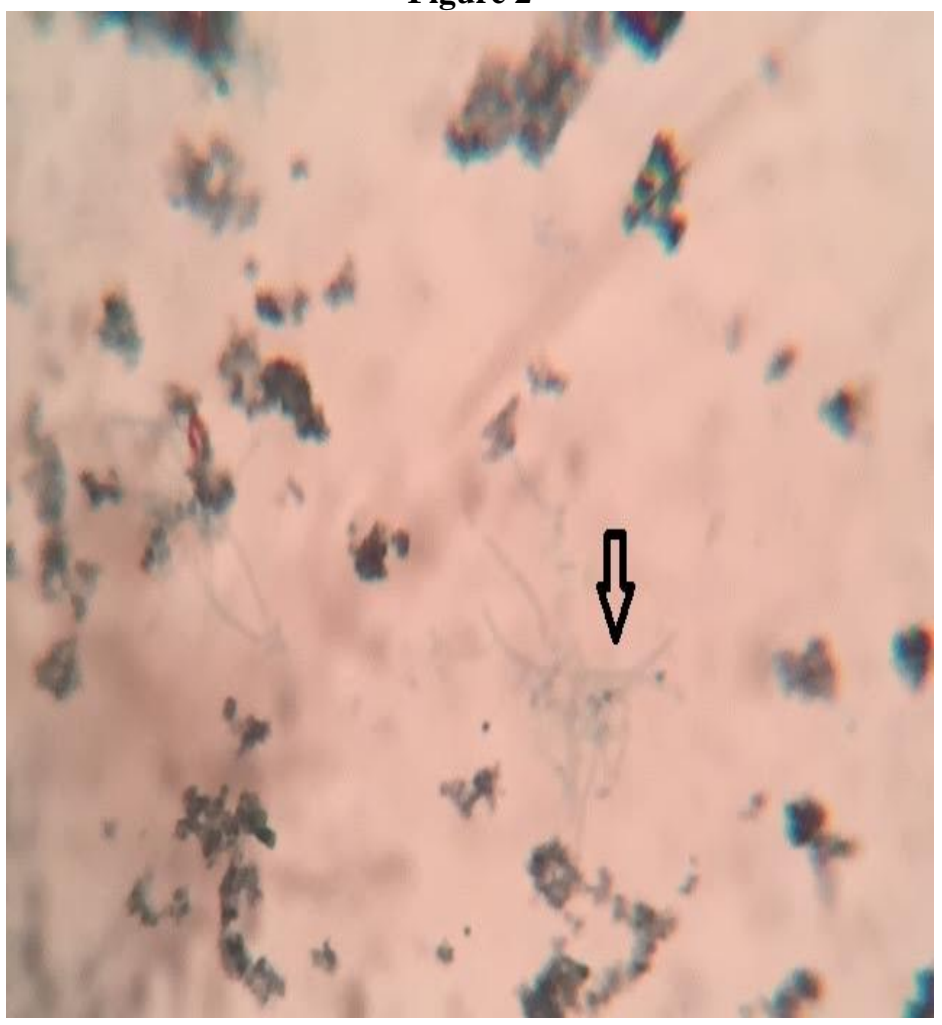

